\title{
Effect of saddle height on skin temperature measured in different days of cycling
}

\author{
Jose Ignacio Priego Quesada 1,2,3* ${ }^{\text {CD, Felipe P. Carpes }}{ }^{3}$, Rosario Salvador Palmer ${ }^{2}$, Pedro Pérez-Soriano \\ and Rosa Ma Cibrián Ortiz de Anda ${ }^{2}$
}

\begin{abstract}
Infrared thermography can be useful to explore the effects of exercise on neuromuscular function. During cycling, it could be used to investigate the effects of saddle height on thermoregulation. The aim of this study was to examine whether different cycling postures, elicited by different knee flexion angles, could influence skin temperature. Furthermore, we also determined whether the reproducibility of thermal measurements in response to cycling differed in the body regions affected or not affected by saddle height. Sixteen cyclists participated in three tests of 45 min of cycling at their individual $50 \%$ peak power output. Each test was performed in a different knee flexion position on the bicycle $\left(20^{\circ}, 30^{\circ}, 40^{\circ}\right.$ knee flexion when the pedal crank was at $\left.180^{\circ}\right)$. Different knee angles were obtained by changing saddle height. Skin temperatures were determined by infrared thermography before, immediately after and 10 min after the cycling test, in 16 different regions of interest (ROI) in the trunk and lower limbs. Changes in saddle height did not result in changes in skin temperature in the ROI. However, lower knee flexion elicited higher temperature in popliteus after cycling than higher flexion ( $p=0.008$ and $E S=0.8$ ), and higher knee flexion elicited lower temperature variation in the tibialis anterior than intermediate knee flexion $(p=0.004$ and $E S=0.8)$. Absolute temperatures obtained good and very good intraday reproducibility in the different measurements (ICCs between 0.44 and 0.85 ), but temperature variations showed lower reproducibility (ICCs between 0.11 and 0.74 ). Different postures assumed by the cyclist due to different saddle height did not influence temperature measurements. Skin temperature can be measured on different days with good repeatability, but temperature variations can be more sensitive to the effects of an intervention.
\end{abstract}

Keywords: Infrared thermography, Thermal imaging, Exercise, Bike-fit, Reproducibility, Reliability

\section{Background}

Bike-fit represents the adjustment of the geometry of the bicycle and its components according to the dimensions of the cyclist body, with the general purpose of maximizing performance and reducing injury risk (Disley and Li 2014; Fonda et al. 2014). Saddle height is one of the most frequently assessed dimensions during bike-fit (Bini et al. 2011).

\footnotetext{
*Correspondence: j.priego.gibd@gmail.com

${ }^{2}$ Biophysics and Medical Physics Group, Department of Physiology,

University of Valencia, Avd/Blasco Ibañez 15, 46010 Valencia, Spain

Full list of author information is available at the end of the article
}

Different studies showed that changes in saddle height affect neuromuscular activation during cycling. Sanderson and Amoroso (2009) observed that greater knee flexion (differences of $17^{\circ}$ ) due to low saddle height decreased neuromuscular activation in the soleus and medial gastrocnemius. Jorge and Hull (1986) found lower activation of quadriceps and hamstring when saddle height was set at $95 \%$ of trochanteric length compared to saddle height set at $100 \%$. These differences in the neuromuscular activation can theoretically affect the heat production of the muscles, thus affecting skin temperature (Saltin et al. 1970; Taylor 2000; Kenny et al. 2003). Indeed, these thermal effects could be even greater during aerobic cycling in hot environments, resulting in high skin temperatures that reduce the heat dissipation between the core and the

\section{垈 Springer}

(C) 2016 Priego Quesada et al. This article is distributed under the terms of the Creative Commons Attribution 4.0 International License (http://creativecommons.org/licenses/by/4.0/), which permits unrestricted use, distribution, and reproduction in any medium, provided you give appropriate credit to the original author(s) and the source, provide a link to the Creative Commons license, and indicate if changes were made. 
skin (Sawka et al. 2012). This could increase core temperature to such high values $\left(\sim 40{ }^{\circ} \mathrm{C}\right)$ as to lead to fatigue and an increased risk of heat exhaustion and heat stroke (Nybo 2010; Sawka et al. 2012; Best et al. 2014).

The effects of the saddle height during cycling have been extensively examined through assessment of kinematics (Ferrer-Roca et al. 2012; Fonda et al. 2014), electromyography (Jorge and Hull 1986; Sanderson and Amoroso 2009), pedal forces (Iriberri et al. 2008; Bini et al. 2014), and gas exchange (Peveler and Green 2011). Considering the previously discussed relationship between muscle activation and heat production (Saltin et al. 1970; Kenny et al. 2003; Priego Quesada et al. 2015a), infrared thermography (IRT) can be an additional tool to explore the effects of saddle height during cycling from a thermal point of view. IRT is a technique that enables measurement of skin temperature, with valuable applications in the study of the thermal effects of physical exercise (Formenti et al. 2013; Abate et al. 2013). This technique provides some advantages to skin temperature measurement over other methods (e.g. thermocouples) since it is a non-invasive and non-contact technique with high sensitivity and accuracy (de Andrade Fernandes et al. 2014). However, to properly measure skin temperature it is necessary to control intervening factors (for details, see Fernández-Cuevas et al. 2015) in order to reduce variability. Variability of skin temperature data on different days can be greater than the effect of changes in saddle height on skin temperature. In addition, reproducibility can be lower in ROIs affected by different saddle heights. For this reason, when research is focused on exploring different conditions on different days, such as in the present study, it is important to consider the reproducibility of skin temperature. McCoy et al. (2011) observed excellent reproducibility between days for IRT images from the paraspinal region. However, Zaproudina et al. (2008) found moderate reproducibility in the trunk and poor reproducibility in the extremities. The authors suggested that this result was probably due to physiological variability of blood flow in the distal parts of the body (Zaproudina et al. 2008). These studies were performed under baseline conditions but reproducibility data after exercise is still necessary.

Here we set out to examine the influence of different cycling postures elicited by different knee flexion angles on skin temperature in response to cycling exercise. A secondary purpose was to determine the reproducibility of thermal measurements taken for specific regions of interest (ROI) after exercise performed on three different days. Different studies have shown differences in neuromuscular activation in quadriceps, hamstrings and gastrocnemius due to changes in saddle height (Jorge and Hull 1986; Sanderson and Amoroso 2009). For this reason, we hypothesized that higher knee flexion would reduce the skin temperature in the calf and in the hamstrings due a possible lower neuromuscular activation, and increase temperature in the quadriceps, due to possible higher neuromuscular activation, as reflected in the literature. Secondly, it was hypothesized that reproducibility on different days would be very good, so allowing a similar thermal baseline state, and that after-test reproducibility would not be affected by changes in saddle height (e.g. ROI of the trunk). However, poor reproducibility could be expected after exercise in the ROI affected by changes in the saddle height (calf, hamstrings and quadriceps).

\section{Methods}

\section{Participants}

Sixteen male cyclists categorized as club level, in accordance with the recommendations of Ansley and Cangley (2009), participated in this study. Mean (standard deviation): age [29 (10) years], body mass [77 (9) kg], height [178.7 $(6.5) \mathrm{cm}$ ], average cycling training volume [230 (133) $\mathrm{km} /$ week], peak power output [273 (48) Watts]. Of the sixteen cyclists, one had a preferred left limb according to Waterloo Footedness Questionnaire (Elias et al. 1998). They all gave their written informed consent before participating. The study procedures complied with the Declaration of Helsinki, and were approved by the University's ethics committee (approval number H1384344515519).

In order to measure the temperature of the participants' skin under similar conditions, all of them were informed that they should not drink alcohol or smoke for at least $12 \mathrm{~h}$ before the test, should not carry out highintensity or exhaustive exercise for at least $24 \mathrm{~h}$ before each test, and should avoid drinking coffee or other stimulants, avoid wearing any jewellery, sunbathing or being exposed to UV rays, as well as refraining from using sunscreen/sun blockers, body lotions and creams before the test. They should not eat for at least $2 \mathrm{~h}$ before the test. Finally, each participant was measured at the same time in the three tests performed in order to reduce the intrasubject effect of the circadian cycle.

\section{Protocol}

The participants completed one pre-test and three main tests carried out on different days. The differences between the main tests were the knee flexion and extension amplitudes. All trials were performed on a stationary cycle ergometer (Cardgirus Medical, Bikemarc, Sabadell, Spain).

In the first visit, all participants performed an incremental cycling trial into determine maximal power output. This incremental trial consisted of 5-min warm-up 
at initial workload of $50 \mathrm{~W}$, followed by sequential 1-min phases in which the workload was increased in steps of $25 \mathrm{~W}$ until exhaustion (Carpes et al. 2011). The pedaling cadence was controlled at $90 \pm 3 \mathrm{rpm}$, and exhaustion was defined as the moment when the cyclist was no longer able to maintain a pedaling cadence of $87 \mathrm{rpm}$. Peak power output $\left(\mathrm{PO}_{\max }\right)$ was defined as the workload of the last phase completed. Posture and the saddle height during the incremental test were set at the same as on their own bicycles.

The three main tests began with a 3-min warm-up at $50 \mathrm{~W}$ and $90 \mathrm{rpm}$. Firstly, the participants stood in underpants for $10 \mathrm{~min}$ in order to adapt to the thermal room temperature before the IRT measurements. After this, participants put on their cycling clothes and shoes (same in the three tests). The participants then cycled for $45 \mathrm{~min}$ at $50 \%$ POmax at $90 \pm 2 \mathrm{rpm}$ while maintaining a specific cycling posture. Each main test was performed with a specific knee flexion angle $\left(40^{\circ}, 30^{\circ}\right.$, or $\left.20^{\circ}\right)$ when the pedal crank was at $180^{\circ}$, and the order of the tests was randomized. The knee angle was defined as the angle of knee flexion relative to the anatomical reference posture (static upright standing posture) taken as zero degrees (offset posture) (Peveler et al. 2012). Trunk flexion (maintained between $40^{\circ}$ and $50^{\circ}$ between the transverse plane and the union of the left acromion and the olecranon tuberosity), arm extension (maintained as a $75^{\circ}-90^{\circ}$ angle between the arms and the trunk), and the horizontal posture of the saddle, as defined by the plummet method (Zani 2010), were controlled throughout the tests. Environmental conditions during the test with the knee flexion angle at $40^{\circ}, 30^{\circ}$ and $20^{\circ}$ were $23.4 \pm 1.1^{\circ} \mathrm{C}$ and $45.4 \pm 12.5 \%, 23.6 \pm 1.2{ }^{\circ} \mathrm{C}$ and $40.7 \pm 11.3 \%$, and $24.0 \pm 1.2{ }^{\circ} \mathrm{C}$ and $50.8 \pm 11.2 \%$ relative humidity, respectively.

Posture was determined before each main test. Participants cycled at $50 \mathrm{~W}$ and $90 \mathrm{rpm}$, with knee flexion angle (obtained by changing the saddle height) using a 2D kinematic analysis system (IBV, Valencia, Spain) with a high-definition video camera sampling at $50 \mathrm{~Hz}$ (Sony Handycam HDR-FX1, Sony Corp., Tokyo, Japan). Reflective markers were attached to the lateral malleolus, lateral femoral condyle, greater trochanter, left acromion, and olecranon tuberosity from the left body side. A correction factor consisting of adding $2.2^{\circ}$ to the measurements was performed (Fonda et al. 2014).

\section{Skin temperature measurement}

Skin temperatures were determined in the main tests by IRT camera with infrared resolution of $320 \times 240$ pixels, thermal sensitivity $<0.05^{\circ} \mathrm{C}$, and accuracy of $\pm 2{ }^{\circ} \mathrm{C}$ (FLIR E-60, Flir Systems Inc., Wilsonville, Oregon, USA). A black body (BX-500 IR Infrared Calibrator, CEM, Shenzhen,
China) was used to ensure a correct calibration of the camera. Thermal images of each participant were taken three times: before the cycling test and after $10 \mathrm{~min}$ of thermal adaptation to room temperature (Marins et al. 2014), immediately after the cycling test, and $10 \mathrm{~min}$ after finishing the cycling test. The images were recorded while the participant was standing up wearing underpants. With the aim of avoiding the effect of skin surface rubbing on skin measurement, sweat was not removed after the cycling test. The camera was positioned $1 \mathrm{~m}$ away from the participant and kept perpendicular to the body regions of interest.

Environmental conditions were controlled (e.g. lighting and temperature controlled room, no person apart from the investigator and the participant and no electronic equipment within a range of 5 meters). An antireflective panel was placed behind the participant to minimize effects from infrared radiation reflected in the wall (Hildebrandt et al. 2012). For all measurements, air temperature and relative humidity were measured using a thermo-hygrometer (Digital thermo-hygrometer, TFA Dostmann, Wertheim-Reicholzheim, Germany) and were computerized in the camera settings.

Sixteen ROIs were defined (chest, abdomen, upper back, lower back, vastus lateralis, rectus femoris, abductor, vastus medialis, biceps femoris, semitendinosus, knee, popliteal, tibialis anterior, gastrocnemius, ankle anterior, and Achilles) (Fig. 1). Each ROI of a similar area was selected for all participants and for each time measurement. For the lower limbs, ROIs were selected in the preferred limb. Absolute mean temperature of each ROI was obtained using thermography software (Thermacam Researcher Pro 2.10 software, FLIR, Wilsonville, Oregon, USA), with an emissivity factor of 0.98 (Steketee 1973).

Skin temperature variation was assessed for each ROI based on the following variables (Priego Quesada et al. 2015a):

- $\Delta \mathrm{T}$ : Difference between temperature before and immediately after the cycling test, expressed in ${ }^{\circ} \mathrm{C}$.

- $\Delta$ T10: Difference between temperature before and 10 min after the cycling test, expressed in ${ }^{\circ} \mathrm{C}$.

- $\Delta$ Tafter: Difference between temperature immediately after and $10 \mathrm{~min}$ after the cycling test, expressed in ${ }^{\circ} \mathrm{C}$.

\section{Statistical analysis}

The statistics package SPSS 21 (SPSS Statistics, IBM) was used for the statistical analysis. The normality of each variable was confirmed by the Shapiro-Wilk test ( $p>0.05$ ). After this, differences between the three knee flexions $\left(40^{\circ}, 30^{\circ}\right.$, and $\left.20^{\circ}\right)$ for absolute temperatures in 


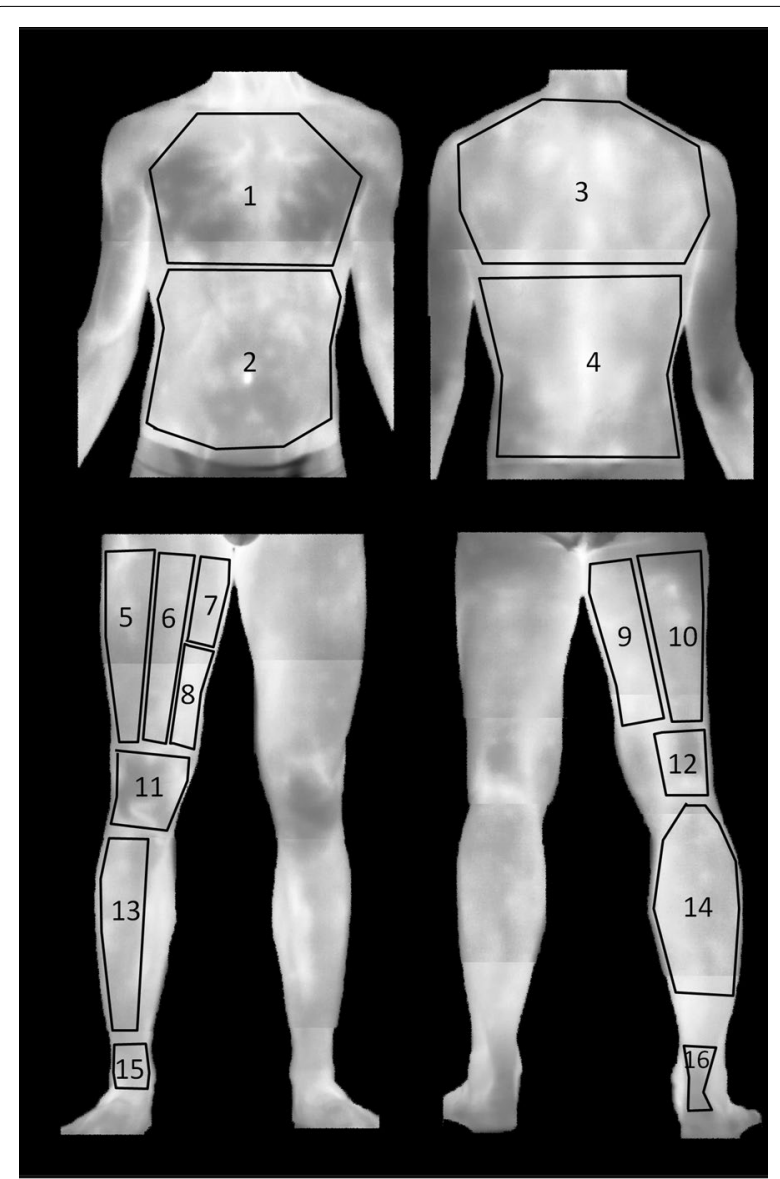

Fig. 1 Representative illustration of the regions of interest (ROI): 1 chest, 2 abdomen, 3 upper back, 4 lower back, 5 vastus lateralis, 6 rectus femoris, 7 abductor, 8 vastus medialis, 9 biceps femoris, 10 semitendinosus, 11 knee, 12 popliteal, 13 tibialis anterior, 14 gastrocnemius, 15 ankle anterior, and 16 Achilles

each ROI were examined by applying two-way repeated measures ANOVA. For absolute temperatures, one-way repeated measures ANOVA were performed in each ROI. For both analyses, Bonferroni post hoc tests were used for pairwise comparison if applicable. Intra-class correlation coefficient (ICC) from model "2,1" (Shrout and Fleiss 1979) was calculated to determine the intraday reproducibility of each ROI. To assess reproducibility, the following classification of ICC values was used (Weir 2005): values 1.00-0.81 (excellent reproducibility), $0.80-0.61$ (very good), $0.60-0.41$ (good), $0.40-0.21$ (reasonable) and, from $0.20-0.00$ (poor). The typical error of the measurement was calculated to represent absolute consistency across the tests (Hopkins 2000; Weir 2005). Data are reported as mean \pm SD with $95 \%$ confidence intervals (95\% CI). Effect sizes (ES) (Cohen 1988) were calculated using a purpose-designed Excel spreadsheet (Microsoft Inc., USA). The level of statistical significance was taken to be $\mathrm{p}<0.05$ and ES $>0.8$ (Cohen 1988).

\section{Results}

\section{Effects of knee angle on skin temperature}

Table 1 shows the temperature values obtained in each ROI corresponding to the different knee angle during cycling. Participants performed the three cycling tests at a specific knee flexion $\left(40^{\circ}, 30^{\circ}\right.$ and $\left.20^{\circ}\right)$ calculated from the offset posture. The absolute knee angle values for $40^{\circ}$, $30^{\circ}$ and $20^{\circ}$ were $50.4 \pm 3.5^{\circ}, 39.8 \pm 4.0$, and $30.0 \pm 4.9$, respectively.

In the analysis of the absolute temperatures in the ROI, differences were obtained only in the popliteus (Table 1). Immediately after the cycling test, knee flexion at $20^{\circ}$ elicited higher temperature in popliteus than knee flexion at $40^{\circ}\left(20^{\circ}\right.$ vs $40^{\circ}: 32.2 \pm 0.7$ vs $31.6 \pm 0.7{ }^{\circ} \mathrm{C}$, $\mathrm{p}=0.008$ and $\mathrm{ES}=0.8,95 \% \mathrm{CI}$ of the difference between conditions $\left[0.1,0.9{ }^{\circ} \mathrm{C}\right]$ ). No differences were obtained for absolute temperatures between the three flexion angles in the other fifteen ROIs ( $p>0.05$ and $\mathrm{ES}<0.8$ ).

In the temperature variation analysis, differences were observed only in the tibialis anterior (Table 2). Knee flexion at $30^{\circ}$ elicited higher $\Delta \mathrm{T} 10$ in the tibialis anterior than knee flexion at $20^{\circ}\left(30^{\circ}\right.$ vs $20^{\circ}: 0.3 \pm 0.9$ vs $-0.2 \pm 0.8^{\circ} \mathrm{C}$, $\mathrm{p}=0.004$ and $\mathrm{ES}=0.8,95 \% \mathrm{CI}$ of the difference between conditions $\left[0.2,1.1{ }^{\circ} \mathrm{C}\right]$ ). No differences were found between the three flexion angles for temperature variation in the other fifteen ROIs ( $p>0.05$ and $E S<0.8$ ).

\section{Intraday reproducibility}

Table 1 shows the intra-class correlation coefficients of the absolute temperatures obtained from the three tests. Before the cycling test, the different ROIs presented good and very good reproducibility. Immediately after cycling, the different ROIs continued showing good and very good reproducibility. Moreover, the ROI of the knee showed excellent reproducibility. Ten minutes after the cycling test all ROIs presented ICC values higher than 0.6 (very good reliability).

Table 2 shows the temperature variations and their intra-class correlation coefficients. The ROI of the trunk presented good and very good reproducibility in the three temperature variations. However, the ROIs of the lower limbs presented lower values of ICC in temperature variations than in absolute values. $\Delta \mathrm{T}$ values presented, in some ROIs, poor (rectus femoris) and reasonable reproducibility (abductor, biceps femoris, and popliteus). This tendency increased for $\Delta \mathrm{T} 10$ values, more ROIs showing poor (vastus lateralis, rectus femoris, abductor and popliteus) and reasonable reproducibility (vastus medialis, biceps femoris and gastrocnemius). In the $\Delta$ Tafter values, almost all ROIs of the limbs presented good reproducibility, and only three presented reasonable ICC values (popliteus, gastrocnemius and ankle anterior). 


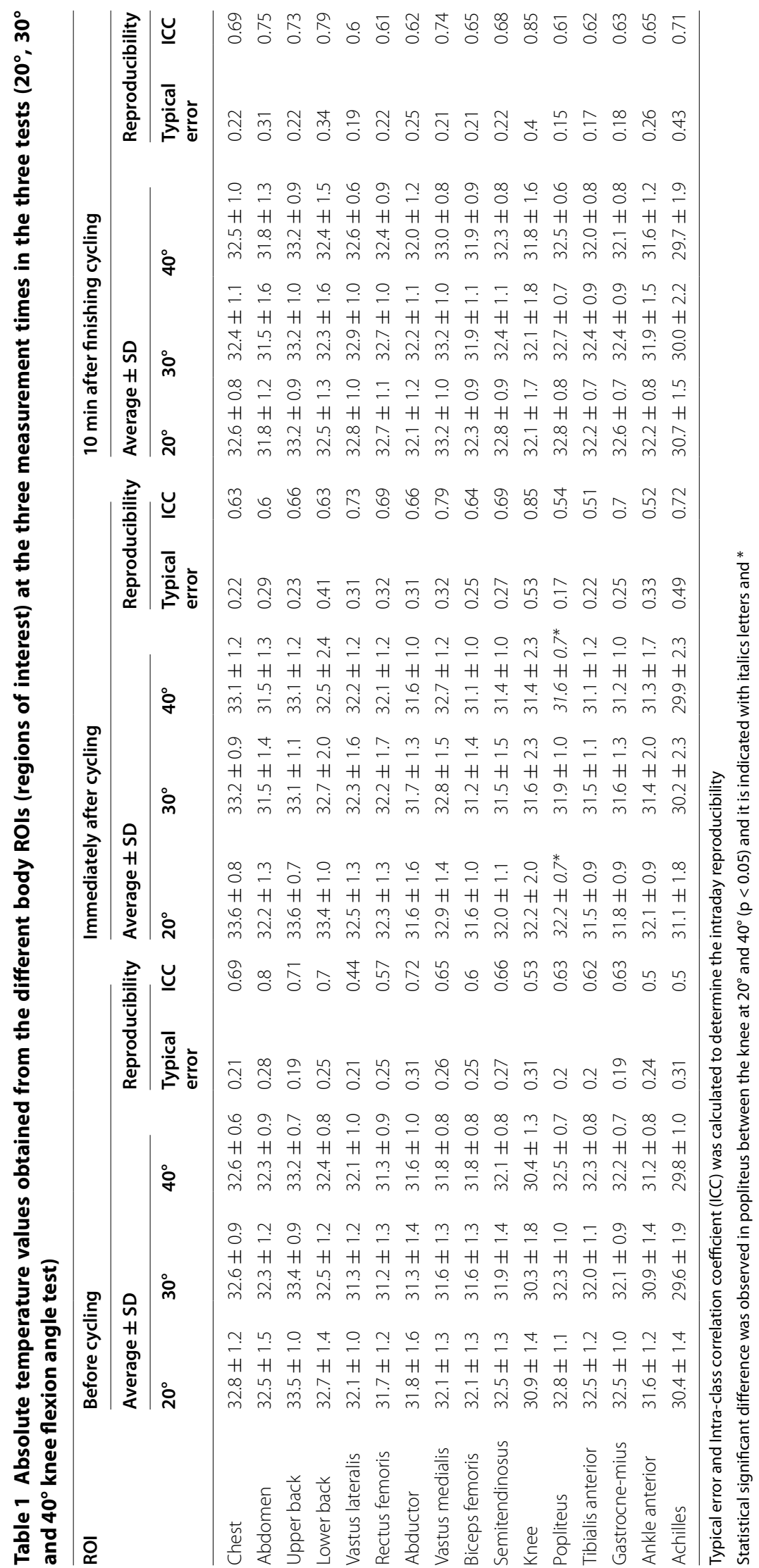




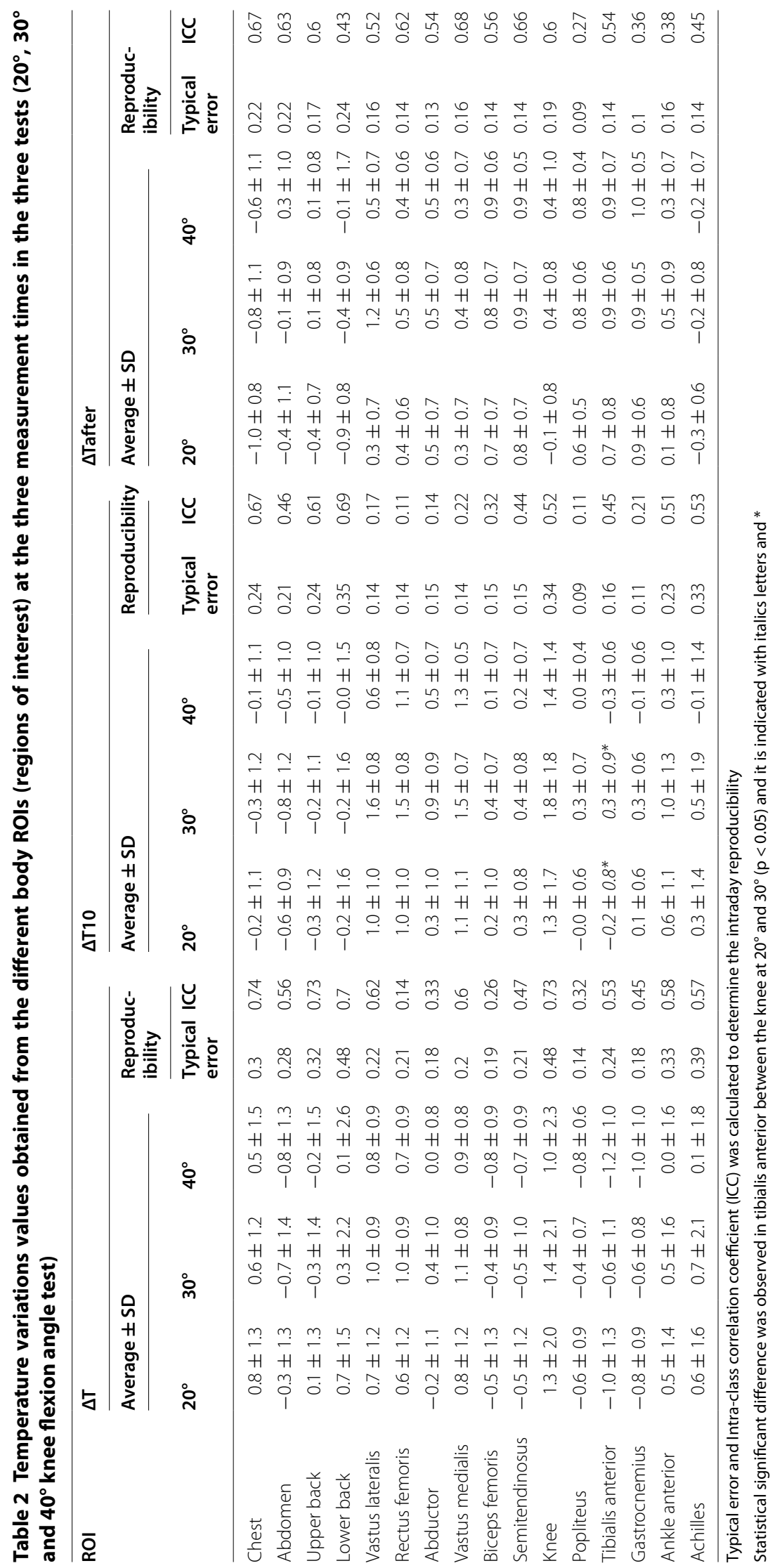




\section{Discussion}

The purpose of the present study was to analyse the effect of knee flexion (elicited by different saddle heights) on skin temperature after cycling, and also to determine the reproducibility of skin temperature taken at specific ROI in the three different tests performed by cyclists. The main findings were that the ROI with possible changes in neuromuscular activation produced by the saddle height did not affect skin temperature. However, greatest knee extension presented higher temperature in the popliteus after cycling than the greatest knee flexion, and the greatest knee flexion elicited lower temperature variation $(\Delta \mathrm{T} 10)$ in the tibialis anterior than intermediate knee flexion. Furthermore, absolute temperatures obtained good and very good reproducibility at the different measurement times, but temperature variations of the lower limbs presented lower reproducibility.

We hypothesized the effects of different knee angles and saddle height positions on skin temperature, but no differences were observed. Although changes in the saddle height can increase the neuromuscular activation of specific muscles and thus increase their heat production (Saltin et al. 1970; Kenny et al. 2003), these were not reflected in increased skin temperature, probably due to higher sweat rate (Buono et al. 2010; Fujii et al. 2014). Higher sweat rate reduces the skin temperature and this favours its thermal gradient with the core (Cuddy et al. 2014). Thus, the result of the skin temperature is the balance between metabolic heat production and heat dissipation (González-Alonso 2012).

Thermal effects produced by the different knee flexions were observed in poplitius and tibialis anterior. The greatest knee extension $\left(20^{\circ}\right.$ when the pedal crank is at $180^{\circ}$ ) showed a higher temperature in popliteus (ranged from 0.1 to $0.9^{\circ} \mathrm{C}$ ) than in the greatest knee flexion $\left(40^{\circ}\right)$. Different authors have associated pain in popliteus with a too high saddle height (Callaghan 2005; Silberman 2013). It is possible that the higher tendon elongation produced by the greatest knee extension results in a higher tendon blood volume (Kubo et al. 2009), and then an increase in skin temperature (Hildebrandt et al. 2012). On the other hand, the greatest knee flexion $\left(40^{\circ}\right)$ presented lower temperature variation $\left(\Delta \mathrm{T} 10\right.$, ranged between 0.2 and $\left.1.1{ }^{\circ} \mathrm{C}\right)$ in the tibialis anterior than intermediate knee flexion $\left(30^{\circ}\right)$. Tibialis anterior is an ankle stabilizer during pedaling (So et al. 2005). A recent study observed lower range of ankle motion in an optimal saddle height $\left(25^{\circ}\right)$ than in low saddle height $\left(45^{\circ}\right)$ (Bini et al. 2014). These results were in agreement with the present study, in which the lowest increase in the tibialis anterior skin temperature was found between the greatest $\left(40^{\circ}\right)$ and the intermediate knee flexion $\left(30^{\circ}\right)$. Lower range of ankle motion can result in lower muscular activation of tibialis anterior (So et al. 2005), resulting in a lower temperature variation. However, although higher differences between the greatest and the lowest knee flexion $\left(20^{\circ}\right)$ could be expected, no differences were found between both postures. Further studies are necessary to explain and to validate the thermal differences between knee flexion in the popliteus and tibialis anterior.

The intraday reproducibility results obtained before cycling test in absolute temperature were better, except in the ROI of the knee, than those presented by Zaproudina et al. (2008). They presented a 0.76 ICC value for the trunk anterior $(0.69$ and 0.80 in chest and abdomen in the present study), 0.32 in the back (0.71 and 0.70 in upper and lower back) 0.42 in the thigh (0.60) 0.76 in the knee (0.53), and 0.52 in the calf (0.63). Differences between the results may be due to a number of reasons: differences in the ROI identification, different room adaptation (10 $\mathrm{min}$ lower in the present study) or/and differences in the variability of the blood flow of the participants (Zaproudina et al. 2008). In any case, IRT measurement has presented good and very good reproducibility following the rigorous methodology used by different authors and organizations (Ring and Ammer 2000; Ammer 2008; Hildebrandt et al. 2012; Priego Quesada et al. 2015a). Furthermore, the present study showed that reproducibility is still good after exercise. However, the temperature variation analysis showed similar reproducibility in the trunk, but worse in the lower limbs. This may be due to the fact that temperature variations can be more sensitive to changes in the saddle height than absolute temperatures. Hence, different studies have shown significant temperature variations after their interventions, but no differences in absolute temperatures (Formenti et al. 2013; Priego Quesada et al. 2015b). Similarly, different studies focus their significant results on temperature variations, probably because absolute temperatures do not reflect the effect of their interventions (Bertmaring et al. 2008; Chudecka and Lubkowska 2010). Further studies should investigate the reproducibility of temperature data after exercise without any intervention and whether temperature variations of the lower limbs present higher intraday reproducibility without changes in the saddle height or another type of intervention.

One limitation of the present study was that neuromuscular activation was not measured during the cycling test. Surface electromyography could show differences in the neuromuscular activation due to different saddle heights as reported by the literature (Jorge and Hull 1986; Sanderson and Amoroso 2009).

Sweat on the skin surface after the cycling tests could have influenced the IRT data and it should also be considered a limitation of the present study. A film of water 
on the skin may work as a filter for infrared radiation and, therefore, it could lead to an underestimation of the thermal data of the infrared measures (Ammer 2009). However, the studies undertaken are insufficient to clarify the effect of sweat on skin emissivity in infrared measures. For this reason, it was decided not to remove the sweat on the skin for the thermographic measurements after the cycling tests in order to avoid an increase of skin temperature due to rubbing, and also the reduction of the natural process of sweat evaporation (Priego Quesada et al. 2015b).

The present study assessed the effects on skin temperature after cycling $45 \mathrm{~min}$ at $50 \%$ of the POmax. This intensity was chosen with the aim of carrying out a moderate aerobic intensity test. Future studies could explore if cycling tests with higher workload, but of shorter duration, could increase neuromuscular differences between knee flexions and later skin temperature.

\section{Conclusions}

Different knee flexions, after 45 min of cycling, did not present differences in skin temperature in the ROI linked to changes in the neuromuscular activation reported in the literature. This finding demonstrates that the application of skin temperature analysis using IRT for studying the effects of different saddle heights does not appear to be suitable. However, reproducibility of the absolute temperatures after exercise on different days was good, temperature variations being more sensitive to the effects of an intervention. This means that skin temperature can be measured on different days by IRT, but temperature variation analysis may be better for studying the effect of an intervention. Future studies are needed to validate this last hypothesis.

\section{Authors' contributions}

All the authors contributed in the conception and design of experiments. JIPQ participated in the collection of the data. JPPQ and RMC participated in the analysis of the data, and they, with FC and RSP, participated in the interpretation of the data. All the authors participated in drafting the article or revising it critically for important intellectual content. All authors approved the final version of the manuscript, all persons designated as authors qualify for authorship, and all those who qualify for authorship are listed. All authors read and approved the final manuscript.

\section{Authors' information}

JIPQ Ph.D. student in the Physiology Department of the University of Valencia, with a doctoral fellowship (FPU) received from the Spanish Ministry of Education, Culture and Sport. Thermographer of level 1 by the Infrared Training Center. Researcher of the Research Group in Sport Biomechanics (GIBD). Previously he worked for 2 years with a research grant in the Biomechanics Institute of Valencia (IBV). His research topics of interest are the use of infrared thermography in sport assessment, cycling biomechanics, sport biomechanics and footwear assessment. FC is a full professor in Federal University of Pampa in Brazil. Dr. C's research focuses on developing a basic understanding of the neuromechanics of rhythmic movements during standing, walking and cycling and applying this information to sports and rehabilitation, considering experimental models in human and other animals. RSP graduated in Physics from the University of Valencia, Spain, in 1978 and received her PhD in Physics from the same university in 1987. She is currently professor of Biophysics and Medical Physic in the Department of Physiology, director of the Master's Degree in Physiology and Coordinator of the Doctoral Programme in Physiology of the University of Valencia. Her current research is focused on non-lesive medical image techniques such as structured light and thermography and digital speckle procedures. She is member of different scientific associations and Member of the Organizing Committee of some Scientific Congresses. PPS Ph.D. Professor at Department of Physical Education and Sport (University of Valencia, Spain). He co-manages the research group "GIBD" (Research Group in Sport Biomechanics) with projects funded by public/private institutions. Most of his research projects are linked to sports biomechanics, physical activity, particularly related to footwear and foot orthotics. He has several national and international publications with impact journals under "Sport Sciencies" category. He has also directed three books related to sports biomechanics and various chapters related to the same area. RMC received M.Sc. and Ph.D. degrees in Physics from the University of Valencia, Spain, in 1978 and 1987, respectively. She is currently professor of Biophysics in the Department of Physiology and director of the Master's Degree in Medical Physics. She was Head of that Department during 3 years. She has been involved in several projects related to image and pattern analysis applied to Medicine and Sports. She has 4 research sexenis. She is a member of different scientific associations and a Member of the Organizing Committee of some Scientific Congresses.

\section{Author details}

${ }^{1}$ Research Group in Sport Biomechanics (GIBD), Department of Physical Education and Sports, University of Valencia, Valencia, Spain. ${ }^{2}$ Biophysics and Medical Physics Group, Department of Physiology, University of Valencia, Avd/Blasco Ibañez 15, 46010 Valencia, Spain. ${ }^{3}$ Applied Neuromechanics Group, Laboratory of Neuromechanics, Federal University of Pampa, Uruguaiana, RS, Brazil.

\section{Acknowledgements}

This research was funded with doctoral fellowship (FPU) received from the Spanish Ministry of Education, Culture and Sport. The authors are grateful to Servei d'Esports of the Universidad Politécnica de Valencia for the loan of the cycle ergometer. Thanks are given for all participants who volunteered for this study.

\section{Competing interests}

The authors declare that they have no competing interests.

Received: 24 July 2015 Accepted: 15 February 2016

Published online: 27 February 2016

\section{References}

Abate M, Di Carlo L, Di Donato L et al (2013) Comparison of cutaneous termic response to a standardised warm up in trained and untrained individuals. J Sports Med Phys Fitness 53:209-215

Ammer K (2008) The Glamorgan Protocol for recording and evaluation of thermal images of the human body. Thermol Int 18:125-144

Ammer K (2009) Does neuromuscular thermography record nothing else but an infrared sympathetic skin response? Thermol Int 19:107-108

Ansley L, Cangley P (2009) Determinants of "optimal" cadence during cycling. Eur J Sport Sci 9:61-85

Bertmaring I, Babski-Reeves K, Nussbaum MA (2008) Infrared imaging of the anterior deltoid during overhead static exertions. Ergonomics 51:16061619. doi:10.1080/00140130802216933

Best S, Thompson M, Caillaud C et al (2014) Exercise-heat acclimation in young and older trained cyclists. J Sci Med Sport Sports Med Aust 17:677-682. doi:10.1016/j.jsams.2013.10.243

Bini MR, Hume PA, Croft JL (2011) Effects of bicycle saddle height on knee injury risk and cycling performance. Sports Med 41:463-476

Bini RR, Hume PA, Kilding AE (2014) Saddle height effects on pedal forces, joint mechanical work and kinematics of cyclists and triathletes. Eur J Sport Sci 14:44-52. doi:10.1080/17461391.2012.725105

Buono MJ, Lee NVL, Miller PW (2010) The relationship between exercise intensity and the sweat lactate excretion rate. J Physiol Sci 60:103-107. doi:10.1007/s12576-009-0073-3 
Callaghan MJ (2005) Lower body problems and injury in cycling. J Bodyw Mov Ther 9:226-236

Carpes FP, Diefenthaeler F, Bini RR et al (2011) Influence of leg preference on bilateral muscle activation during cycling. J Sports Sci 29:151-159

Chudecka M, Lubkowska A (2010) Temperature changes of selected body's surfaces of handball players in the course of training estimated by thermovision, and the study of the impact of physiological and morphological factors on the skin temperature. J Therm Biol 35:379-385

Cohen J (1988) Statistical power analysis for the behavioral sciences. Lawrence Erlbaum, London

Cuddy JS, Hailes WS, Ruby BC (2014) A reduced core to skin temperature gradient, not a critical core temperature, affects aerobic capacity in the heat. J Therm Biol 43:7-12. doi:10.1016/j.jtherbio.2014.04.002

de Andrade Fernandes A, dos Santos Amorim PR, Brito CJ et al (2014) Measuring skin temperature before, during and after exercise: a comparison of thermocouples and infrared thermography. Physiol Meas 35:189

Disley BX, Li F-X (2014) Metabolic and kinematic effects of self-selected Q Factor during bike fit. Res Sports Med 22:12-22. doi:10.1080/15438627.2 013.852093

Elias LJ, Bryden MP, Bulman-Fleming MB (1998) Footedness is a better predictor than is handedness of emotional lateralization. Neuropsychologia 36:37-43

Fernández-Cuevas I, Bouzas Marins JC, Arnáiz Lastras J et al (2015) Classification of factors influencing the use of infrared thermography in humans: a review. Infrared Phys Technol 71:28-55. doi:10.1016/j.infrared.2015.02.007

Ferrer-Roca V, Roig A, Galilea P, García-López J (2012) Influence of saddle height on lower limb kinematics in well-trained cyclists: static vs. dynamic evaluation in bike fitting. J Strength Cond Res 26:3025-3029

Fonda B, Sarabon N, Li F-X (2014) Validity and reliability of different kinematics methods used for bike fitting. J Sports Sci 32:940-946

Formenti D, Ludwig N, Gargano M et al (2013) Thermal imaging of exerciseassociated skin temperature changes in trained and untrained female subjects. Ann Biomed Eng 41:863-871. doi:10.1007/s10439-012-0718-x

Fuji N, Honda Y, Komura Ket al (2014) Effect of voluntary hypocapnic hyperventilation on the relationship between core temperature and heat loss responses in exercising humans. J Appl Physiol Bethesda Md 1985 117:1317-1324. doi:10.1152/japplphysiol.00334.2014

González-Alonso J (2012) Human thermoregulation and the cardiovascular system. Exp Physiol 97:340-346. doi:10.1113/expphysiol.2011.058701

Hildebrandt C, Zeilberger K, Ring EFJ, Raschner C (2012) The application of medical infrared thermography in sports medicine. Ultrasound 10:2

Hopkins WG (2000) Measures of reliability in sports medicine and science. Sports Med Auckl NZ 30:1-15

Iriberri J, Muriel X, Larrazabal I (2008) The bike fit of the road professional cyclist related to anthropometric measurements and the Torque of de Crank (P242). In: Estivalet M, Brisson P (eds) The engineering of sport 7. Springer, Heidelberg. pp 483-488

Jorge M, Hull ML (1986) Analysis of EMG measurements during bicycle pedalling. J Biomech 19:683-694

Kenny GP, Reardon FD, Zaleski W et al (2003) Muscle temperature transients before, during, and after exercise measured using an intramuscular multisensor probe. J Appl Physiol Bethesda Md 1985 94:2350-2357. doi:10.1152/japplphysiol.01107.2002
Kubo K, Ikebukuro T, Yaeshima K et al (2009) Effects of static and dynamic training on the stiffness and blood volume of tendon in vivo. J Appl Physiol 106:412-417. doi:10.1152/japplphysiol.91381.2008

Marins JCB, Moreira DG, Cano SP et al (2014) Time required to stabilize thermographic images at rest. Infrared Phys Technol 65:30-35. doi:10.1016/j. infrared.2014.02.008

McCoy M, Campbell I, Stone P et al (2011) Intra-examiner and inter-examiner reproducibility of paraspinal thermography. PLoS One 6:e16535. doi:10.1371/journal.pone.0016535

Nybo L (2010) Cycling in the heat: performance perspectives and cerebral challenges. Scand J Med Sci Sports 20(Suppl 3):71-79. doi:10.1111/j.1600-0838.2010.01211.x

Peveler WW, Green JM (2011) Effects of saddle height on economy and anaerobic power in well-trained cyclists. J Strength Cond Res Natl Strength Cond Assoc 25:629-633. doi:10.1519/JSC.0b013e3181d09e60

Peveler WW, Shew B, Johnson S, Palmer TG (2012) A kinematic comparison of alterations to knee and ankle angles from resting measures to active pedaling during a graded exercise protocol. J Strength Cond Res 26:3004-3009. doi:10.1519/JSC.0b013e318243fdcb

Priego Quesada JI, Carpes FP, Bini RR et al (2015a) Relationship between skin temperature and muscle activation during incremental cycle exercise. J Therm Biol 48:28-35. doi:10.1016/j.jtherbio.2014.12.005

Priego Quesada JI, Lucas-Cuevas AG, Gil-Calvo M et al (2015b) Effects of graduated compression stockings on skin temperature after running. J Therm Biol 52:130-136. doi:10.1016/j.jtherbio.2015.06.005

Ring EFJ, Ammer K (2000) The technique of infrared imaging in medicine. Thermol Int 10:7-14

Saltin B, Gagge AP, Stolwijk JA (1970) Body temperatures and sweating during thermal transients caused by exercise. J Appl Physiol 28:318-327

Sanderson DJ, Amoroso AT (2009) The influence of seat height on the mechanical function of the triceps surae muscles during steady-rate cycling. J Electromyogr Kinesiol 19:e465-e471

Sawka MN, Cheuvront SN, Kenefick RW (2012) High skin temperature and hypohydration impair aerobic performance. Exp Physiol 97:327-332. doi:10.1113/expphysiol.2011.061002

Shrout PE, Fleiss JL (1979) Intraclass correlations: uses in assessing rater reliability. Psychol Bull 86:420-428. doi:10.1037/0033-2909.86.2.420

Silberman MR (2013) Bicycling injuries. Curr Sports Med Rep 12:337-345. doi:10.1249/JSR.0b013e3182a4bab7

So RCH, Ng JK-F, Ng GYF (2005) Muscle recruitment pattern in cycling: a review. Phys Ther Sport 6:89-96. doi:10.1016/j.ptsp.2005.02.004

Steketee J (1973) Spectral emissivity of skin and pericardium. Phys Med Biol 18(5):686-694

Taylor NAS (2000) Principles and practices of heat adaptation. J Hum Environ Syst 4:11-22

Weir JP (2005) Quantifying test-retest reliability using the intraclass correlation coefficient and the SEM. J Strength Cond Res Natl Strength Cond Assoc 19:231-240. doi:10.1519/15184.1

Zani Z (2010) Pedalear bien/While Pedaling. Ediciones Tutor SA, Madrid

Zaproudina N, Varmavuo V, Airaksinen O, Närhi M (2008) Reproducibility of infrared thermography measurements in healthy individuals. Physiol Meas 29:515. doi:10.1088/0967-3334/29/4/007

\section{Submit your manuscript to a SpringerOpen ${ }^{\circ}$ journal and benefit from:}

- Convenient online submission

- Rigorous peer review

- Immediate publication on acceptance

- Open access: articles freely available online

- High visibility within the field

- Retaining the copyright to your article

Submit your next manuscript at $>$ springeropen.com 